\title{
A blend of 3 mushrooms dose-dependently increases butyrate production by
} the gut microbiota

\author{
J. Verhoeven ${ }^{1}$, D. Keller ${ }^{2}$, S. Verbruggen ${ }^{1}$, K. Youssef Abboud ${ }^{1}$ and K. Venema ${ }^{1^{*}}$ \\ ${ }^{1}$ Centre for Healthy Eating \& Food Innovation (HEFI), Maastricht University - campus Venlo, Villafloraweg 1, \\ 5928 SZ Venlo, the Netherlands; ${ }^{2}$ Keller Consulting Group, 2417 Beachwood Blvd., Beachwood, OH 44122, USA; \\ k.venema@maastrichtuniversity.nl
}

Received: 6 February 2021 / Accepted: 7 September 2021

(c) 2021 Wageningen Academic Publishers

OPEN ACCESS (C)(1)(2) RESEARCH ARTICLE

\begin{abstract}
The gut microbiota has been indicated to play a crucial role in health and disease. Apart from changes in composition between healthy individuals and those with a disease or disorder, it has become clear that also microbial activity is important for health. For instance, butyrate has been proven to be beneficial for health, because, amongst others, it is a substrate for the colonocytes, and modulates the host's immune system and metabolism. Here, we studied the effect of a blend of three mushrooms (Ganoderma lucidum GL AM P-38, Grifola frondosa GF AM P36 and Pleurotus ostreatus PO AM-GP37)) on gut microbiota composition and activity in a validated, dynamic, computercontrolled in vitro model of the colon (TIM-2). Predigested mushroom blend at three doses $(0.5,1.0$ and $1.5 \mathrm{~g} / \mathrm{day}$ of ingested mushroom blend) was fed to a pooled microbiota of healthy adults for $72 \mathrm{~h}$, and samples were taken every day for microbiota composition (sequencing of amplicons of the V3-V4 region of the 16S rRNA gene) and activity (short-chain fatty acid (SCFA) production). The butyrate producing genera Lachnospiraceae UCG-004, Lachnoclostridium, Ruminococcaceae UCG-002 and Ruminococcaceae NK4A214-group are all dose-dependently increased when the mushroom blend was fed. Entirely in line with the increase of these butyrate-producers, the cumulative amount of butyrate also dose-dependently increased, to roughly twice the amount compared to the control (medium without mushroom blend) on the high-dose mushroom blend. Butyrate proportionally made up $53.1 \%$ of the total SCFA upon feeding the high-dose mushroom blend, compared to $27 \%$ on the control medium. In conclusion, the (polysaccharides in the) mushroom blend led to substantial increase in butyrate by the gut microbiota. These results warrant future mechanistic research on the mushroom blend, as butyrate is considered to be one of the microbial metabolites that contributes to health, by increasing barrier function and modulating inflammation.
\end{abstract}

Keywords: Ganoderma lucidum, Grifola frondosa, Pleurotus ostreatus, in vitro colon model, SCFA, butyrate

\section{Introduction}

The gut microbiota, containing trillions of bacteria, archaea, fungi and yeasts, and bacteriophages and viruses, has been shown over the past decades to play a crucial role in health and disease (Matijasic et al., 2020; Vemuri et al., 2020). Diseases and disorders affected by the gut microbiota range from those occurring in the gastrointestinal (GI) tract, where these micro-organisms reside, to diseases elsewhere in the body. The diseases of the GI tract include inflammatory bowel disease (IBD) (Lavelle and Sokol, 2020), irritable bowel syndrome (IBS)
(Collins, 2014) and colorectal cancer (CRC) (Fan et al., 2021). Systemic diseases and disorders include, amongst others, allergy of skin (Petersen et al., 2019) and lungs (Barcik et al., 2020), obesity and associated cardiovascular diseases (Marzullo et al., 2020), type I diabetes (Verduci et al., 2020) and other autoimmune diseases (Reyes-Castillo et al., 2021), and even phenomena related to the brain, such as brain development and cognition (Almeida et al., 2020), Parkinson's and Alzheimer's disease (Ceppa et al., 2020), and autism spectrum disorders (MacFabe, 2015). Although the full spectrum of mechanisms by which the gut microbiota interacts with the host are still being discovered, 
it is clear that the microorganisms influence our immune system and metabolism (Backhed et al., 2004; Geurts et al., 2014), through e.g. interacting directly with immune cells, modulating gene expression in a variety of tissue, and/or production of metabolites which are absorbed through the gut epithelium and transported to distant organs through blood and lymph systems.

One of the metabolites produced by the gut microbiota that has received a lot of attention is butyrate. Butyrate is one of the short-chain fatty acids (SCFA), the others being acetate and propionate, which are organic acids produced within the lumen of the colon by the gut microbiota mainly through fermentation of undigested/indigestible dietary carbohydrates (Blaak et al., 2020). Undigested/indigestible dietary and endogenous protein, such as the protein-part of mucus, and sloughed epithelial cells, can be fermented into SCFA and branched-chain fatty acids (BCFA) (Topping and Clifton, 2001). Butyrate is considered to be healthpromoting, as it has been shown to have anti-carcinogenic and anti-inflammatory effects, and it strengthens the gut barrier function (Hamer et al., 2008). Its production from carbohydrate fermentation is thought to be more important for health than from protein fermentation, as protein fermentation also leads to various putrefactive metabolites, which are considered to be toxic to the host (Macfarlane and Macfarlane, 2012).

A prebiotic is defined as 'a selectively fermented ingredient that results in specific changes in the composition and/or activity of the gastrointestinal microbiota, thus conferring benefit(s) upon host health' (Gibson et al., 2017). Where in the past 'prebiotic' was synonymous to 'bifidogenic' (increase in Bifidobacterium) effects (Gibson and Roberfroid, 1995), the latest definition also allows room for health benefits related to microbial activity (amongst other production of SCFA).

Mushrooms have been the subject of intense research due to their bioactive components. Medicinal mushrooms have been used for centuries in Asia, originating in China, and are currently still applied as traditional Chinese medicine (TCM) (Martel et al., 2017; Zhou et al., 2019). Mushrooms produce a large number of pharmaceutically active proteins, which have become popular sources of natural antitumor, antimicrobial or immunoenhancing agents (Zhou et al., 2019). Moreover, polysaccharides and some small-molecule components, such as flavones, other polyphenols and terpenes, are present in mushrooms, and these may interact with the (immune system of the) host or its gut microbiota (Jayachandran et al., 2017). Carbohydrates are the major components in mushrooms, accounting from 35 to $70 \%$ of the dry weight, with variations in different species. Among these are polysaccharides, such as $\beta$-glucans, consisting primarily of $\beta-(1 \rightarrow 3)$ linkages with some $\beta-(1 \rightarrow 6)$ branches, as well as hetero-polysaccharides containing mannose, galactose, fucose, xylose and rhamnose. Mushroom polysaccharides have been shown to possess a variety of therapeutic and gut microbiota modulation benefits (Cheung, 2013; Ruthes et al., 2016; Sameer Kumar et al., 2016), e.g. the $\beta$-glucans are also known to promote butyrogenic bacteria in the colon, thus producing butyrate (Friedman, 2016; Han et al., 2020; Jayachandran et al., 2018).

In vivo, it is quite difficult, if not impossible, to disentangle the direct effects that the mushroom bioactives have on the immune system and those occurring through the gut microbiota (such as butyrate production). Effects on the gut microbiota are therefore usually tested in vitro, using models that mimic the colon. One of these models is the TNO in vitro model of the proximal colon (TIM2) (Minekus et al., 1999), which is a validated, dynamic, computer-controlled system that accurately mimics host physiology in the colon with respect to temperature, $\mathrm{pH}$, removal of microbial metabolites (mimicking absorption through the gut epithelium) and presence of a dense, active gut microbiota (Venema, 2015). It has been used extensively to test the effect of prebiotics, potential novel prebiotics and dietary carbohydrates on gut microbiota composition and activity (Larsen et al., 2019; Martina et al., 2019; Miguez et al., 2020a,b; Sayago-Ayerdi et al., 2019; Venema et al., 2020).

The aim of the study described here was to investigate the potential prebiotic effect of a blend of three mushrooms by investigating the effect on composition and activity of the gut microbiota. This was studied using the validated TNO dynamic in vitro model of the colon (TIM-2). Changes in composition (using sequencing of the V3-V4 region of the $16 \mathrm{~S}$ rRNA gene) and activity (SCFA production) of the gut microbiota of healthy adults upon feeding three different doses of the mushroom blend were tested.

\section{Materials and methods}

\section{Characteristics of the mushroom blend}

A proprietary blend of three mushrooms (Ganoderma lucidum GL AM P-38, Grifola frondosa GF AM P36 and Pleurotus ostreatus PO AM-GP37) was provided by Aloha Medicinals (Carson City, NV, USA) as a fine coarse powder.

\section{Predigestion of the mushroom blend}

To remove digestible components and components that would normally be absorbed in the small intestine, the mushroom blend was predigested in bulk. This protocol was adapted from Brodkorp et al. (2019) and Minekus et al. (2014). However, it included dialysing the digestion products using a dialysis unit that is incorporated in TNO in vitro model of the stomach and small intestine (TIM1) (Minekus et al., 1995). This unique dialysis membrane (Sureflux 07 L, Nipro Europe, Zaventem, Belgium) removes 
digestion products (and water) and prevents them from reaching the colon (where they would normally also not arrive). After predigestion, this slurry was freeze dried and ground to a fine particle size, to get a homogeneous powder, which was used in subsequent fermentation experiments in TIM-2.

\section{TNO's in vitro model of the colon (TIM-2)}

TIM-2 (Supplementary Figure S1) simulates the large intestine or colon, consisting of four interconnected compartments containing flexible membranes with a volume of $125 \mathrm{ml}$. By applying pressure on the flexible membrane, peristaltic movements are accomplished (Venema, 2015). Average conditions of healthy human individuals were simulated including: body temperature $\left(37^{\circ} \mathrm{C}\right), \mathrm{pH}$ in the lumen ( $\left.\mathrm{pH} 5.9\right)$, composition and rate of secretion fluids, delivery of a predigested substrate, mixing and transport of intestinal contents by peristalsis, absorption of water and microbial metabolites through the means of a dialysis system, and presence of a complex, high density, metabolically active and anaerobic microbiota of human healthy adults as described in detail before (Venema, 2015; Venema et al., 2020). The dialysis system is a crucial component of the model as it prevents accumulation of microbial metabolites, which would otherwise inhibit or even kill the members of the gut microbiota. The model was inoculated with a standardised microbiota of healthy adult human volunteers (Venema et al., 2000). For this, faeces were collected from healthy adult volunteers from the department ( $n=6$; 3 male, 3 female; average age $25.8 \pm 3.2$ years) and pooled in an anaerobic cabinet to allow for a standardised microbiota (Venema et al., 2000) that could be used throughout the experiments, and allowed for comparison between runs. We have shown before, when studying carbohydrate fermentation, that pooling the microbiota from different individuals leads to a pool with the same metabolic capacity as observed in the individual samples (Aguirre et al., 2014), likely due to high functional redundancy in metabolic capacity of members of the gut microbiota (Moya and Ferrer, 2016; Thursby and Juge, 2017), especially with respect to carbohydrate fermentation (Aguirre et al., 2014). The pooled microbiota was aliquoted, snap frozen in liquid nitrogen and stored at $-80{ }^{\circ} \mathrm{C}$ until inoculation in the model. Four units were run in parallel. After an adaptation period of $16 \mathrm{~h}$, in which the inoculum adapted to the conditions in the system (Venema et al., 2000), the different doses of the substrates were fed to the microbiota over a period of 3 days through the feeding syringe.

\section{Experimental set-up}

Three doses of predigested mushroom blend were tested, corresponding to $0.5 \mathrm{~g} /$ day, $1 \mathrm{~g} /$ day and $1.5 \mathrm{~g} /$ day of ingestion. The predigested blend was added to the standard medium used in TIM-2 (standard ileal efflux medium - SIEM; Supplementary Table S1). The predigested mushroom blend was added to the standard carbohydrates used in the control medium (SIEM). This medium was originally developed by Gibson et al. (1988) and mimics the complex undigestible carbohydrates (pectin, xylan, arabinogalactan, amylopectin and resistant starch) on an average Western diet.

\section{Sampling}

Samples were taken every day for a period of three days from both the lumen and the dialysate of the system (at 0, 24,48 and $72 \mathrm{~h}$ ). Samples from both the lumen and dialysate were measured for SCFA concentrations. The production of metabolites at the moment of addition of the substrates was artificially set to zero, and cumulative production of the different SCFAs was calculated from that moment onwards. Samples from the lumen of the model were analysed on composition of the microbiota by sequencing the V3 - V4 region of the $16 \mathrm{~S}$ rRNA gene using Illumina sequencing as indicated below.

\section{Short chain fatty acid analyses}

Both lumen $(1.5 \mathrm{ml})$ and dialysate $(2 \mathrm{ml})$ samples were centrifuged at $14,000 \times g$ for $10 \mathrm{~min}$. Fifty $\mu \mathrm{l}$ (lumen) or 150 $\mu \mathrm{l}$ (dialysate) samples were mixed with $650 \mu \mathrm{l}$ (lumen) or $550 \mu \mathrm{l}$ (dialysate) internal standard solution, containing methanol, internal standard ( $2 \mathrm{mg} / \mathrm{ml} 2$-ethyl butyric acid), and formic acid (20\%). The analysis was carried out on a GC-MS (8890 GC System; Agilent Technolgies, Amstelveen, the Netherlands) equipped with a PAL3 RSI 85 autosampler (Agilent) by injecting $1 \mu \mathrm{l}$ sample on a DB-FATWAX Ultra Inert column (30 m, $0.25 \mathrm{~mm}, 0.25$ $\mu \mathrm{m}$ Agilent). The temperature settings of the injector port, oven, flame-ionization detector and mass spectrometer detector were 250, 200, 275 and $225^{\circ} \mathrm{C}$, respectively. The flow rate over the column was $1.2 \mathrm{ml} / \mathrm{min}$.

\section{Illumina MiSeq sequencing of V3-V4 region of the 16S rRNA gene}

Samples for microbiota composition were analysed by sequencing PCR amplicons of the V3-V4 region of the $16 \mathrm{~S}$ rRNA gene. Briefly, the isolation of genomic DNA from the faecal samples ( $1 \mathrm{ml}$ lumen sample) was performed using standard molecular biology kits from ZYMO Research as provided by the manufacturer (BaseClear, Leiden, the Netherlands). The PCR amplification of the V3 and V4 regions, the barcoding and the library preparation were carried out according to established protocols provided by Illumina (Nextera XT DNA Library Preparation Kit and TG Nextera ${ }^{\circledR}$ XT Index Kit v2 Set A, Illumina, Eindhoven, the Netherlands) using the following primers 341F (5'-CCTACGGGNGGCWGCAG-3') and the 785R 
(5'-GACTACHVGGGTATCTAATCC-3') appended with Illumina adaptor sequences. The sequencing was carried out using the Illumina MiSeq system (MiSeq Reagent Kit v3, Illumina) and later the sequences were converted into FASTQ files using the BCL2FASTQ pipeline version 1.8.3. The quality cut was applied based on the Phred quality score. Quantitative Insights Into Microbial Ecology 2 (QIIME 2) software package (2019.4) was used for microbial analyses (Caporaso et al., 2010; Estaki et al., 2020). The sequences were classified using Greengenes (version 13.8) as a reference 16S rRNA gene database.

\section{Statistical analyses}

Correlations between Operational Taxonomic Units (OTUs) and test-products were investigated using the non-parametric Kruskal-Wallis test, by using the software package R (3.5.3) (R Core Team, 2013) in RStudio. Multiple comparison was corrected using the Benjamini-Hochberg false discovery rate (FDR), and $q$-values (FDR-adjusted $P$-values) were considered significantly different at a cut off of $<0.1$.

\section{Results and discussion}

\section{Predigested material}

After predigestion, most of the protein from the mushroom blend was removed. Protein that was left was mostly coming from the digestive enzymes added to predigest the blend (data not shown). The material, from which digestible material was removed through dialysis using a membrane used in hospital for kidney-failure patients, primarily contained carbohydrates ( $\beta$-glucans and heteropolysaccharides; see below) and material attached to cell-wall structures, such as polyphenols, tannins, etc. For the purpose of the current study the latter were not quantified. Recovery of predigested material was $65 \%$ of the material that was initially added to the predigestion, and the material contained $90 \%$ carbohydrates (w/w), and $5 \%$ protein $(\mathrm{w} / \mathrm{w})$ (data not shown).

\section{TIM-2 set-up}

To study the potential prebiotic effects of the blend of three mushrooms, the effect of the blend on gut microbiota composition and activity was evaluated in TIM-2. As most carbohydrates are fermented in the proximal part of the colon, the model mimicked the conditions in the ascending colon. After an overnight adaptation period, in which the SIEM (Cuevas-Tena et al., 2019) was fed, the predigested mushroom blend was fed to the standardised microbiota in TIM-2 at 0 (SIEM only), $0.5 \mathrm{~g} / \mathrm{day}, 1.0 \mathrm{~g} / \mathrm{day}$ and $1.5 \mathrm{~g} /$ day for a period of 3 days. The blend was added to SIEM. Each day samples were taken from the lumen and dialysate of
TIM-2 and changes in microbiota composition and activity were measured.

\section{Changes in microbiota composition}

Changes in composition of the microbiota were evaluated by sequencing amplicons of the V3-V4 region of the $16 \mathrm{~S}$ rRNA gene. Since a standardised microbiota was used, all experiments started with the same composition at time point zero, as can be seen by the close clustering in the weighted UniFrac principal coordinate analysis (Supplementary Figure S2A), a measure for beta-diversity. Over time, the units fed with the mushroom blend moved away from those fed with SIEM only in the weighted UniFrac (Supplementary Figure 2B) indicating changes in composition at the genus level (see below). There were no differences at phylum level (Supplementary Figure S3).

Using the non-parametric Kruskal-Wallis test, we studied which operational taxonomic units (OTUs), at the level of bacterial genera, were different between treatments. This was done at the level of the individual 'interventions' (each dose of mushroom blend separately), as well as grouping the three doses together and looking at the level of 'substrate' (control vs mushroom blend). After correction for multiple comparison, we apply a strict cut-off for significance. For $q$-values $<0.1$ (the corrected $P$-value after correction for multiple comparisons using false-discovery rate) we consider the difference to be significant, and for a $q$-value between 0.2 and 0.1 as a trend. Figure $1 \mathrm{~A}-\mathrm{E}$ shows the OTUs that are significantly different when looking at the individual doses. The plots with OTU names in green show a $q$-value $<0.1$, while those with names in orange have a $q$-value between 0.1 and 0.2 . The latter are shown as well, because some of these become significant when all three doses of the mushroom blend are grouped together (Figure 1F-I). The data show a significant difference for Pediococcus when tested at the individual doses, which can be attributed to its increase at the highest dose of mushroom blend tested. If all doses are grouped together, the significance is lost (data not shown). The other OTUs, Lachnospiraceae UCG004, Lachnoclostridium and the two Ruminococcaceae (UCG-002 and NK4A214-group) are all dose-dependently increased when the mushroom blend is fed (significant for Lachnospiraceae UCG-004 and Ruminococcaceae UCG002 ; trend for the others). This is interesting, because these OTUs are well-known butyrate producers. When examining the difference at the level of all three doses of mushroom blend combined (Figure 1F-I), the butyrate producing OTUs Lachnoclostridium and Ruminococcaceae NK4A214 also become significant. The boxplots show all time-points (T24, T48 and T72) together. Supplementary Figure S4 shows the development of the relative abundance at the individual time-points for Lachnospiraceae UCG-004, Lachnoclostridium and the two Ruminococcaceae (UCG002 and NK4A214-group). This data shows that these taxa 
(dose-dependently) increase over time compared to the condition with SIEM, where these taxa stay more or less at the same abundance or decrease over time.

\section{Production of microbial metabolites}

Apart from composition of the microbiota, we also studied its activity. The major microbial metabolites that have been implicated in health are the SCFA (acetate, propionate and butyrate). Especially butyrate has attracted attention over the past decades as it has been shown to be the primary substrates for the colonocytes, epithelial cells of the colon, and has been shown to be beneficial in inflammation in the gut, due to its effects on gene-expression in immune and other host cells (Hamer et al., 2008).
Figure 2 shows the cumulative SCFA production when the various interventions are fed to the gut microbiota. The profile on the control medium (SIEM) shows that acetate is the major SCFA produced. In vivo the ratio of acetate:propionate:butyrate is in the order of $60 \%: 20 \%: 20 \%$ (Cummings et al., 1987). For SIEM this is also observed in TIM-2 (Table 1). The cumulative amount of total SCFA produced after the 3 -day experiment is $143.8 \mathrm{mmol}$ (Table 2). No differences in production of BCFA was observed (data not shown).

Strikingly, but entirely in line with the increase in relative abundance of the butyrate producing OTUs in Figure 1, upon feeding the three different doses of the mushroom blend, a dose-dependent increase in butyrate proportion

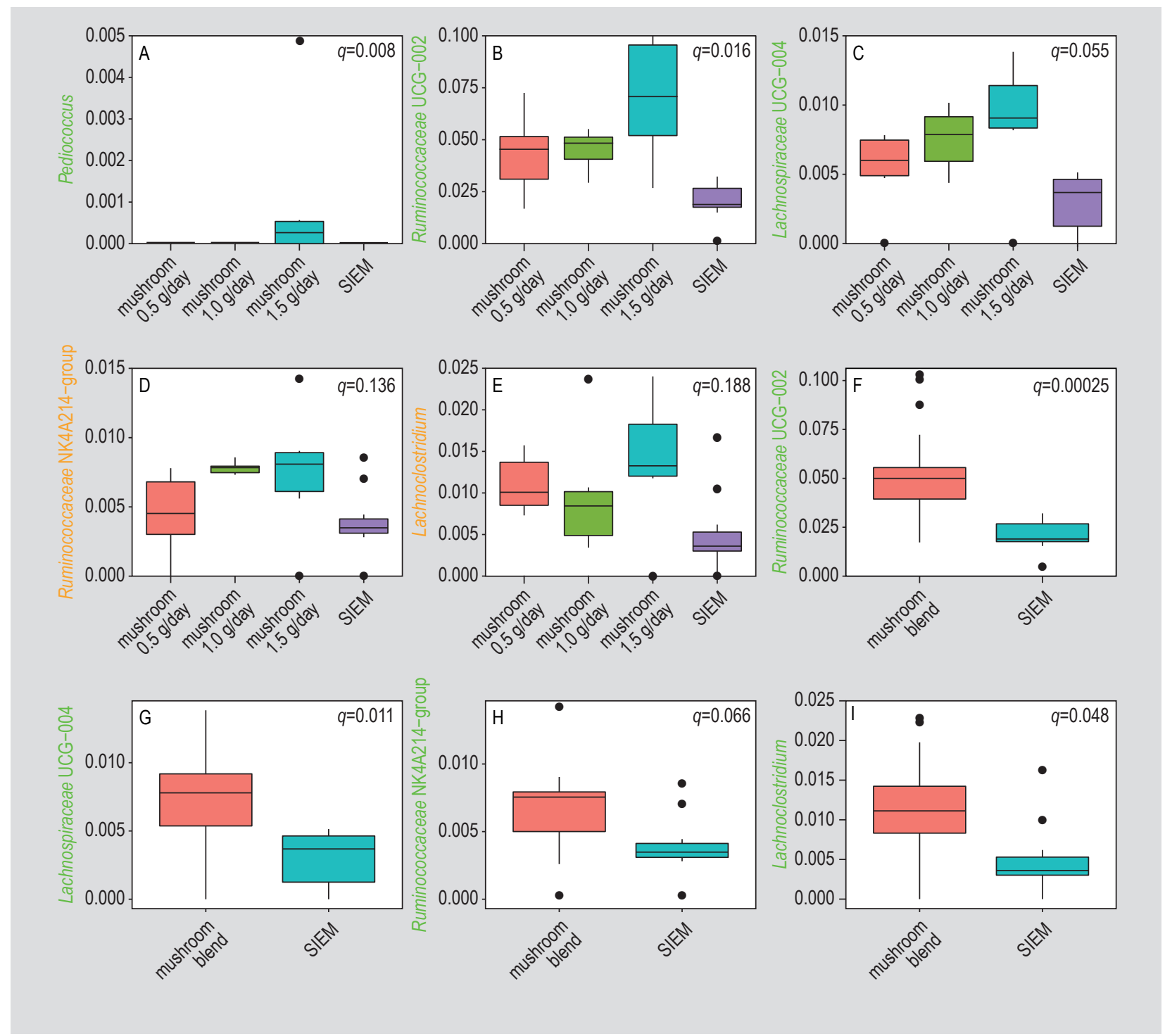

Figure 1. Boxplots of the operational taxonomic units (OTUs) that are different between the different doses and standard ileal efflux medium (SIEM) (A-E) or between the blend (all doses combined) and SIEM (F-I). The $q$-values after false discovery ratecorrection of Krukal-Wallis analysis are provided. A q-value $<0.1$ is considered significant (taxa font in green); one between 0.1 and 0.2 is considered a trend (taxa font in orange). 

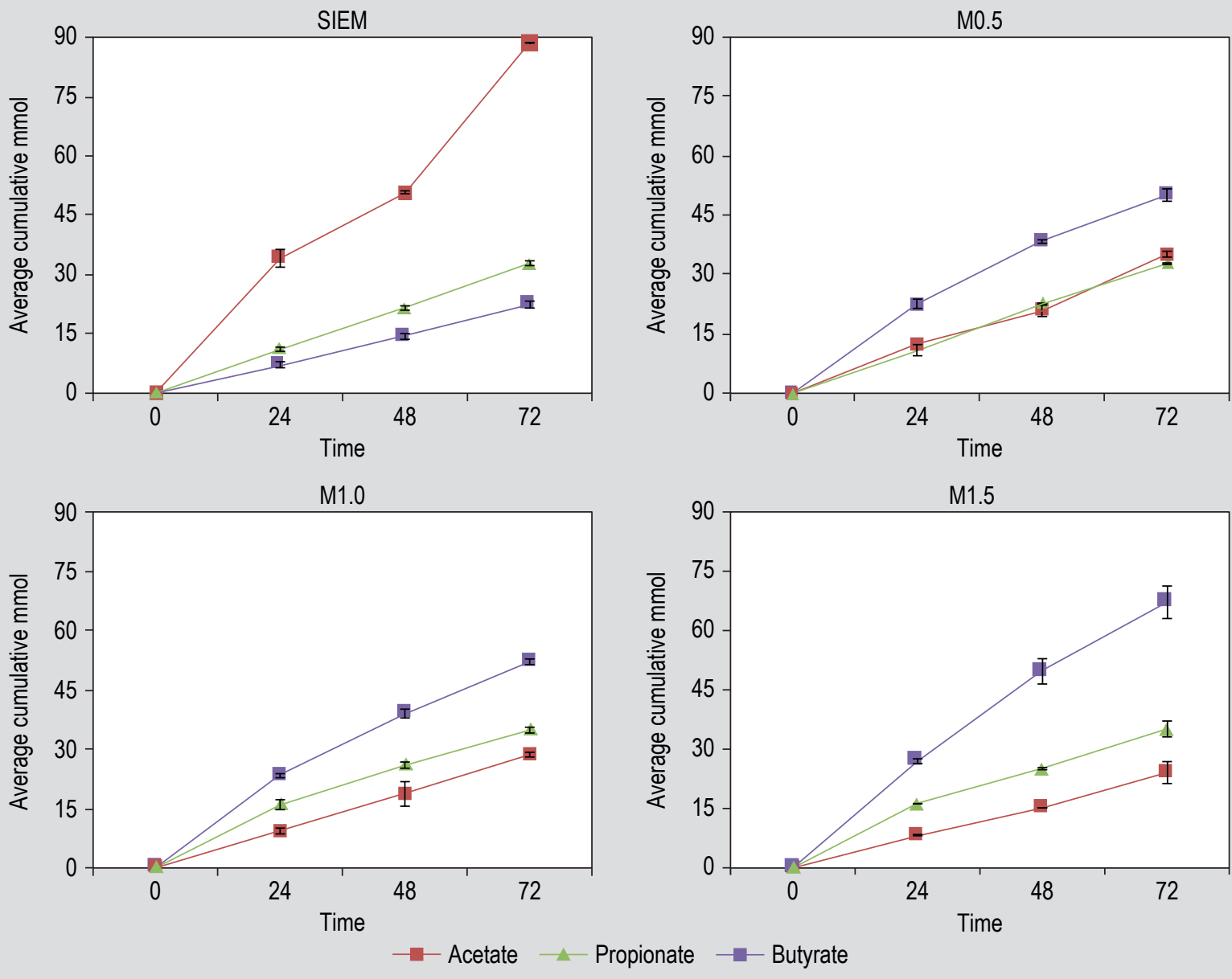

Figure 2. Cumulative production of the short chain fatty acid (SCFA) acetate, propionate and butyrate on the different interventions. M0.5, M1.0 and M1.5: mushroom blend at $0.5 \mathrm{~g} /$ day, $1.0 \mathrm{~g} /$ day and $1.5 \mathrm{~g} / \mathrm{day}$, respectively. SIEM: simulated ileal efflux medium. Error bars indicated range.

Table 1. Ratio of the different short chain fatty acids (SCFA) at time point $72 .^{1}$

\begin{tabular}{llll}
$\begin{array}{l}\text { Intervention/ } \\
\text { metabolite }\end{array}$ & Acetate & Propionate & Butyrate \\
& & & \\
SIEM & $53.3 \pm 0.5 \%$ & $19.8 \pm 0.2 \%$ & $27.0 \pm 0.3 \%$ \\
M0.5 & $29.8 \pm 11.5 \%$ & $27.8 \pm 4.1 \%$ & $42.4 \pm 7.4 \%$ \\
M1.0 & $24.8 \pm 14.4 \%$ & $30.1 \pm 5.3 \%$ & $45.1 \pm 9.1 \%$ \\
M1.5 & $19.1 \pm 8.7 \%$ & $27.8 \pm 4.5 \%$ & $53.1 \pm 14.3 \%$ \\
\hline & & & \\
& \\
&
\end{tabular}

is observed (Table 1). The ratio of butyrate at the lowest dose of the mushroom blend is $42.4 \%$ (compared to $27.0 \%$ of the control medium), and this increases to $45.1 \%$ for the medium dose, and $53.1 \%$ for the high dose. The latter is almost 2-fold that produced on the control medium.
Table 2. Amount of total short chain fatty acids (SCFA) (mmol) and amount of carbon (C) in the microbial metabolites at time point $72 .^{1}$

\begin{tabular}{lll}
$\begin{array}{l}\text { Intervention/sum of } \\
\text { metabolites }\end{array}$ & Total SCFA (mmol) & Amount of $\mathbf{C}$ \\
& & \\
SIEM & $143.8 \pm 1.4$ & $365.3 \pm 3.4$ \\
M0.5 & $118.0 \pm 1.7$ & $369.0 \pm 5.4$ \\
M1.0 & $115.6 \pm 2.0$ & $370.3 \pm 6.3$ \\
M1.5 & $126.4 \pm 3.3$ & $422.3 \pm 16.9$ \\
\hline & & \\
&
\end{tabular}

Also, the proportion of propionate increases, but not dosedependently, and is $27-30 \%$. Of course, if the proportions of butyrate and propionate increase, the proportion of acetate has to drop (Table 1). With the changes in proportion of the individual SCFA towards more propionate and butyrate, 
the sum of SCFA produced is reduced (Table 2), from 143.8 mmol at T72 for the control down to $118 \mathrm{mmol}$ for the low dose mushroom blend, 115.6 for the middle dose, and 126.4 for the high dose. Although it seems that on the mushroom blend the production of SCFA is thus lower, this is skewed by the fact that acetate only contains 2 carbonatoms, while butyrate contains 4 (and propionate 3 ). So, for every molecule of butyrate twice the number of carbonatoms are needed than for acetate. If we take that into consideration, then rather than a reduction in amount when expressed as mmol, a small increase is observed when expressed in amount of carbon (C) (Table 2). Of course not all C-atoms end up in SCFA, and the results should not be considered an attempt to make a mass-balance, but we have shown before from ${ }^{13} \mathrm{C}$-labeled substrates that $95 \%$ of the labelled C got incorporated in SCFA (Binsl et al., 2010; de Graaf et al., 2010).

It is unlikely that the almost two-fold increase in butyrate proportion can only be attributed to the increases observed in relative abundance of the four butyrate producing taxa. Collectively, in the high dose mushroom blend, these make up approximately $11 \%$ of the observed OTUs, while this is 7 to $8 \%$ in the other doses. Although it is possible that these taxa are modulated by the mushroom blend to such an extent that their metabolism is geared primarily towards butyrate production, it is likely that other taxa, although not significantly modulated in their relative abundance, contribute to the observed changes in butyrate production. There are some (non-significant) dose-dependent increases in relative abundance of Ruminococcus gauvreauii-group, Ruminococcaceae UCG-008, Dorea, an uncharacterised genus in Lachnospiraceae, and an uncharacterised genus in Erysipelotrichaceae, that together also account for 2\% (low dose) to $5.6 \%$ (high dose) of the total relative abundance. It is also possible that the metabolism of other butyrate producers is shifted, without affecting their relative abundance. The conclusions drawn here are based on relative abundance. Although in the current experiments we did not quantitatively measure total bacteria, in previous experiments we have shown that the total number of bacteria remain relatively constant in the model, because we removed an appropriate amount of volume from the system daily to simulate passage to the distal colon (or 'going to the bathroom'). The results obtained here are therefore indeed likely the result of a reduction in acetate/ propionate producers as well as an increase in butyrate producers. However, as mentioned above we cannot exclude that the metabolism of certain taxa changed (from acetate/propionate to butyrate), without major shifts in composition. Future studies with ${ }^{13} \mathrm{C}$-labelled substrates, similar to the ones done by us and others before, might shed a light on that (Binsl et al., 2010; de Graaf et al., 2010; Lamichhane et al., 2018).
In general, ruminococci (including Ruminococcaceae UCG-002 and NK4A214-group) are stimulated by dietary fibre (e.g. (De Angelis et al., 2015; Reider et al., 2020)). The Ruminococcus gauvreauii-group has been shown before to be stimulated by fibres (e.g. Long et al., 2020) and is not inhibited by polyphenols (Firrman et al., 2016). In addition, recently it was shown that members of the family Lachnospiraceae are stimulated by dietary fibre, but depending on the source of fibre (Shang et al., 2021). Similarly, Lachnoclostridium has been shown to be stimulated by some dietary fibres (wheat bran and levan; Adamberg et al., 2018; Shang et al., 2020), but not by others (type 2 resistant starch; Zhang et al., 2020). Also Dorea is stimulated by dietary fibre (e.g. Chen et al., 2020a) and polyphenols (e.g. Kilua et al., 2020). Moreover, also Erysipelotrichaceae has been shown to be stimulated by fibre intake (e.g. Lamichhane et al., 2018), and in this study with ${ }^{13} \mathrm{C}$-labelled polydextrose was correlated with increased SCFA production.

Fungi are remarkable for the variety of high-molecularweight polysaccharide structures that they produce, which are found in all parts of the mushroom (e.g. Wachtel-Galor et al., 2011; Yang et al., 2019). Most studies that investigated the effects of medicinal mushrooms on gut microbiota composition and/or activity have used the purified polysaccharides and animal models. Moreover, very few studies have looked at the (potential synergistic) effects of mushroom blends. For instance, the polysaccharide of one of the mushrooms in the blend G. lucidum, has been tested in various rodent models (mice, rats and hamsters) for the effect on gut microbiota modulation, usually with the primary aim to look at immuno-modulation, using polysaccharide purified either from spores or from mycelium (Chen et al., 2020b; Jin et al., 2019; Khan et al., 2019; Su et al., 2018; Tong et al., 2020; Xie et al., 2019). G. lucidum has been shown to contain linear and branched $\beta$-glucans (Liu et al., 2017), including a highly branched $\beta$-glucan found in G. lucidum spores (Wang et al., 2017). Although the main polysaccharide present in G. lucidum is composed of glucose, other monosaccharides were found in varying proportions, such as arabinose, galactose, xylose and mannose, suggesting the presence of polysaccharides with different chemical structures. Heterogalactans composed of rhamnose, galactose and glucose, and side chains composed of glucose have been described (Pan et al., 2012; Ye et al., 2008). Moreover, other isolated fractions, like oil (Wu et al., 2020) and the triterpenoid ganoderic acid A (Guo et al., 2020b) have been shown to modulate the rodent gut microbiota. Similarly, the purified polysaccharide of $G$. frondosa has been shown to modulate the gut microbiota in rodents (mice and rats) in conjunction with beneficial changes in glucose metabolism and lipid metabolism disorders (Chen et al., 2019; Guo et al., 2020a; Li et al., 2019a,b; Pan et al., 2018, 2020). $\beta$-glucans, but also $\alpha$-glucans are known to be the main component of 
G. frondosa, but also a heteropolysaccharide composed of $(1 \rightarrow 6)$ - $\alpha$-D-Galp with O-2 linked residues of $\alpha$-Manp $\alpha$-Fucp have been found (Jayachandran et al., 2018; Ruthes et al., 2016). Regarding the chemical structure of $P$. ostreatus polysaccharides, $\beta$-glucans are the most prevalent, as well as best studied component of $P$. ostreatus. This mushroom was also found to have glucogalactan $\rightarrow 1)-\alpha$-D-Galp- $(\rightarrow 6$ with residues of $\beta$-L-Glcp at non-reducing ends (Ruthes et al., 2016). The effect of $P$. ostreatus polysaccharides on the gut microbiota composition has been tested in piglets and chickens (Adams et al., 2019; Robinson et al., 2018) and on gut microbiota metabolic activity in in vitro batch fermentations using human inocula (Boulaka et al., 2020; Mitsou et al., 2020). Despite the limitations of such batch fermentations, such as accumulations of microbial metabolites, leading to inhibition and/or death of the microbiota, one of these studies found increased proportions of butyrate with feeding of $P$. ostreatus (Mitsou et al., 2020), although the increase was not as evident as observed in our fermentations in a dynamic in vitro model with the blend of mushrooms.

To our knowledge whole mushrooms, let alone blends of different mushrooms, have not been tested frequently. Rather purified fractions, mostly the polysaccharide fraction, have been investigated. In our experiments, in a validated in vitro model of the proximal colon, that closely mimics physiological conditions in humans, and which has been used for three decades in research on gut microbiology, we show that the blend of G. lucidum, $G$. frondosa and P. ostreatus has a beneficial effect on gut microbiota composition and activity. The amount of extract used would equal 5-15 gram of fresh mushrooms (taking on average a water-content of $90 \%$ ). Given that the model is roughly scaled to the in vivo situation, one would expect 5-15 g of mushroom ingestion to have the same effect in vivo. A number of butyrate producing taxa are increased in relative abundance, which is accompanied by an increase in butyrate proportion. As butyrate is considered to be one of the microbial metabolites that contributes to health, by increasing barrier function and modulating inflammation, it would be good to reproduce these results in a clinical trial.

\section{Supplementary material}

Supplementary material can be found online at https://doi. org/10.3920/BM2021.0015

Table S1. Composition of the simulated ileal efflux medium as originally composed by Gibson et al. (1988).

Figure S1. Schematic diagram of the dynamic, multicompartmental TNO in vitro mode of the colon (TIM-2).
Figure S2. Weighted UniFrac of samples coloured by time point and intervention.

Figure S3. Relative abundance of phyla in the different TIM-2 samples treated with $0.5 \mathrm{~g} / \mathrm{day}, 1.0 \mathrm{~g} / \mathrm{day}, 1.5 \mathrm{~g} /$ day mushroom blend, or SIEM.

Figure S4. Changes over time of Lachnoclostridium, Lachnospiraceae UCG-004, Ruminococcaceae NK4A214group and Ruminococcaceae UCG-002 upon feeding of 0.5 $\mathrm{g} /$ day, $1.0 \mathrm{~g} /$ day, $1.5 \mathrm{~g} /$ day of the mushroom blend or SIEM.

\section{References}

Adamberg, K., Adamberg, S., Ernits, K., Larionova, A., Voor, T., Jaagura, M., Visnapuu, T. and Alamae, T., 2018. Composition and metabolism of fecal microbiota from normal and overweight children are differentially affected by melibiose, raffinose and raffinose-derived fructans. Anaerobe 52: 100-110. https://doi. org/10.1016/j.anaerobe.2018.06.009

Adams, S., Che, D., Hailong, J., Zhao, B., Rui, H., Danquah, K. and Qin, G., 2019. Effects of pulverized oyster mushroom (Pleurotus ostreatus) on diarrhea incidence, growth performance, immunity, and microbial composition in piglets. Journal of the Science of Food and Agriculture 99: 3616-3627. https://doi.org/10.1002/jsfa.9582

Aguirre, M., Ramiro-Garcia, J., Koenen, M.E. and Venema, K., 2014. To pool or not to pool? Impact of the use of individual and pooled fecal samples for in vitro fermentation studies. Journal of Microbiological Methods 107: 1-7. https://doi.org/10.1016/j.mimet.2014.08.022

Almeida, C., Oliveira, R., Soares, R. and Barata, P., 2020. Influence of gut microbiota dysbiosis on brain function: a systematic review. Porto Biomedical Journal 5: 1. https://doi.org/10.1097/j. pbj.0000000000000059

Backhed, F., Ding, H., Wang, T., Hooper, L.V., Koh, G.Y., Nagy, A., Semenkovich, C.F. and Gordon, J.I., 2004. The gut microbiota as an environmental factor that regulates fat storage. Proceedings of the National Academy of Sciences of the USA 101: 15718-15723. https://doi.org/10.1073/pnas.0407076101

Barcik, W., Boutin, R.C.T., Sokolowska, M. and Finlay, B.B., 2020. The role of lung and gut microbiota in the pathology of asthma. Immunity 52: 241-255. https://doi.org/10.1016/j.immuni.2020.01.007

Binsl, T.W., De Graaf, A.A., Venema, K., Heringa, J., Maathuis, A., De Waard, P. and Van Beek, J.H., 2010. Measuring non-steadystate metabolic fluxes in starch-converting faecal microbiota in vitro. Beneficial Microbes 1: 391-405. https://doi.org/10.3920/ BM2010.0038

Blaak, E.E., Canfora, E.E., Theis, S., Frost, G., Groen, A.K., Mithieux, G., Nauta, A., Scott, K., Stahl, B., van Harsselaar, J., van Tol, R., Vaughan, E.E. and Verbeke, K., 2020. Short chain fatty acids in human gut and metabolic health. Beneficial Microbes 11: 411-455. https://doi.org/10.3920/BM2020.0057 
Boulaka, A., Christodoulou, P., Vlassopoulou, M., Koutrotsios, G., Bekiaris, G., Zervakis, G.I., Mitsou, E.K., Saxami, G., Kyriacou, A., Zervou, M., Georgiadis, P. and Pletsa, V., 2020. Genoprotective properties and metabolites of beta-glucan-rich edible mushrooms following their in vitro fermentation by human faecal microbiota. Molecules 25: 3554. https://doi.org/10.3390/molecules25153554.

Brodkorb, A., Egger, L., Alminger, M., Alvito, P., Assuncao, R., Ballance, S., Bohn, T., Bourlieu-Lacanal, C., Boutrou, R., Carriere, F., Clemente, A., Corredig, M., Dupont, D., Dufour, C., Edwards, C., Golding, M., Karakaya, S., Kirkhus, B., Le Feunteun, S., Lesmes, U., Macierzanka, A., Mackie, A.R., Martins, C., Marze, S., McClements, D.J., Menard, O., Minekus, M., Portmann, R., Santos, C.N., Souchon, I., Singh, R.P., Vegarud, G.E., Wickham, M.S.J., Weitschies, W. and Recio, I., 2019. INFOGEST static in vitro simulation of gastrointestinal food digestion. Nature Protocols 14: 991-1014. https://doi.org/10.1038/ s41596-018-0119-1

Caporaso, J.G., Kuczynski, J., Stombaugh, J., Bittinger, K., Bushman, F.D., Costello, E.K., Fierer, N., Pena, A.G., Goodrich, J.K., Gordon, J.I., Huttley, G.A., Kelley, S.T., Knights, D., Koenig, J.E., Ley, R.E., Lozupone, C.A., McDonald, D., Muegge, B.D., Pirrung, M., Reeder, J., Sevinsky, J.R., Turnbaugh, P.J., Walters, W.A., Widmann, J., Yatsunenko, T., Zaneveld, J. and Knight, R., 2010. QIIME allows analysis of high-throughput community sequencing data. Nature Methods 7: 335-336. https://doi.org/10.1038/nmeth.f.303

Ceppa, F.A., Izzo, L., Sardelli, L., Raimondi, I., Tunesi, M., Albani, D. and Giordano, C., 2020. Human gut-microbiota interaction in neurodegenerative disorders and current engineered tools for its modeling. Frontiers in Cellular and Infection Microbiology 10: 297. https://doi.org/10.3389/fcimb.2020.00297

Chen, M., Fan, B., Liu, S., Imam, K., Xie, Y., Wen, B. and Xin, F., 2020a. The in vitro effect of fibers with different degrees of polymerization on human gut bacteria. Frontiers in Microbiology 11: 819. https:// doi.org/10.3389/fmicb.2020.00819

Chen, M., Xiao, D., Liu, W., Song, Y., Zou, B., Li, L., Li, P., Cai, Y., Liu, D., Liao, Q. and Xie, Z., 2020b. Intake of Ganoderma lucidum polysaccharides reverses the disturbed gut microbiota and metabolism in type 2 diabetic rats. International Journal of Biological Macromolecules 155: 890-902. https://doi.org/10.1016/j. ijbiomac.2019.11.047

Chen, Y., Liu, D., Wang, D., Lai, S., Zhong, R., Liu, Y., Yang, C., Liu, B., Sarker, M.R. and Zhao, C., 2019. Hypoglycemic activity and gut microbiota regulation of a novel polysaccharide from Grifola frondosa in type 2 diabetic mice. Food and Chemical Toxicology 126: 295-302. https://doi.org/10.1016/j.fct.2019.02.034

Cheung, P.C.K., 2013. Mini-review on edible mushrooms as source of dietary fiber: preparation and health benefits. Food Science and Human Wellness 2: 162-166. https://doi.org/10.1016/j. fshw.2013.08.001

Collins, S.M., 2014. A role for the gut microbiota in IBS. Nature Reviews Gastroenterology and Hepatology 11: 497-505. https:// doi.org/10.1038/nrgastro.2014.40

Cuevas-Tena, M., Alegria, A., Jesús Lagarda, M. and Venema, K., 2019. Impact of plant sterols enrichment dose on gut microbiota from lean and obese subjects using TIM-2 in vitro fermentation model. Journal of Functional Foods 54: 164-174.
Cummings, J.H., Pomare, E.W., Branch, W.J., Naylor, C.P. and Macfarlane, G.T., 1987. Short chain fatty acids in human large intestine, portal, hepatic and venous blood. Gut 28: 1221-1227. https://doi.org/10.1136/gut.28.10.1221

De Angelis, M., Montemurno, E., Vannini, L., Cosola, C., Cavallo, N., Gozzi, G., Maranzano, V., Di Cagno, R., Gobbetti, M. and Gesualdo, L., 2015. Effect of whole-grain barley on the human fecal microbiota and metabolome. Applied and Environmental Microbiology 81: 7945-7956. https://doi.org/10.1128/AEM.02507-15

De Graaf, A.A., Maathuis, A., De Waard, P., Deutz, N.E., Dijkema, C., De Vos, W.M. and Venema, K., 2010. Profiling human gut bacterial metabolism and its kinetics using [U-13C]glucose and NMR. NMR Biomed 23: 2-12. https://doi.org/10.1002/nbm.1418

Estaki, M., Jiang, L., Bokulich, N.A., McDonald, D., Gonzalez, A., Kosciolek, T., Martino, C., Zhu, Q., Birmingham, A., Vazquez-Baeza, Y., Dillon, M.R., Bolyen, E., Caporaso, J.G. and Knight, R., 2020. QIIME 2 enables comprehensive end-to-end analysis of diverse microbiome data and comparative studies with publicly available data. Current Protocols in Bioinformatics 70: e100. https://doi. org/10.1002/cpbi.100

Fan, X., Jin, Y., Chen, G., Ma, X. and Zhang, L., 2021. Gut microbiota dysbiosis drives the development of colorectal cancer. Digestion 102: 508-515. https://doi.org/10.1159/000508328

Firrman, J., Liu, L., Zhang, L., Arango Argoty, G., Wang, M., Tomasula, P., Kobori, M., Pontious, S. and Xiao, W., 2016. The effect of quercetin on genetic expression of the commensal gut microbes Bifidobacterium catenulatum, Enterococcus caccae and Ruminococcus gauvreauii. Anaerobe 42: 130-141. https://doi. org/10.1016/j.anaerobe.2016.10.004

Friedman, M., 2016. Mushroom polysaccharides: chemistry and antiobesity, antidiabetes, anticancer, and antibiotic properties in cells, rodents, and humans. Foods 5: 80. https://doi.org/10.3390/ foods 5040080

Geurts, L., Neyrinck, A.M., Delzenne, N.M., Knauf, C. and Cani, P.D., 2014. Gut microbiota controls adipose tissue expansion, gut barrier and glucose metabolism: novel insights into molecular targets and interventions using prebiotics. Beneficial Microbes 5: 3-17. https:// doi.org/10.3920/BM2012.0065

Gibson, G.R. and Roberfroid, M.B., 1995. Dietary modulation of the human colonic microbiota: introducing the concept of prebiotics. Journal of Nutrition 125: 1401-1412. https://doi.org/10.1093/ jn/125.6.1401

Gibson, G.R., Cummings, J.H. and Macfarlane, G.T., 1988. Use of a three-stage continuous culture system to study the effect of mucin on dissimilatory sulfate reduction and methanogenesis by mixed populations of human gut bacteria. Applied and Environmental Microbiology 54: 2750-2755. https://doi.org/10.1128/ aem.54.11.2750-2755.1988

Gibson, G.R., Hutkins, R., Sanders, M.E., Prescott, S.L., Reimer, R.A., Salminen, S.J., Scott, K., Stanton, C., Swanson, K.S., Cani, P.D., Verbeke, K. and Reid, G., 2017. Expert consensus document: The International Scientific Association for Probiotics and Prebiotics (ISAPP) consensus statement on the definition and scope of prebiotics. Nature Reviews Gastroenterology and Hepatology 14: 491-502. https://doi.org/10.1038/nrgastro.2017.75 
Guo, W.L., Deng, J.C., Pan, Y.Y., Xu, J.X., Hong, J.L., Shi, F.F., Liu, G.L., Qian, M., Bai, W.D., Zhang, W., Liu, B., Zhang, Y.Y., Luo, P.J., Ni, L., Rao, P.F. and Lv, X.C., 2020a. Hypoglycemic and hypolipidemic activities of Grifola frondosa polysaccharides and their relationships with the modulation of intestinal microflora in diabetic mice induced by high-fat diet and streptozotocin. International Journal of Biological Macromolecules 153: 12311240. https://doi.org/10.1016/j.ijbiomac.2019.10.253

Guo, W.L., Guo, J.B., Liu, B.Y., Lu, J.Q., Chen, M., Liu, B., Bai, W.D., Rao, P.F., Ni, L. and Lv, X.C., 2020b. Ganoderic acid A from Ganoderma lucidum ameliorates lipid metabolism and alters gut microbiota composition in hyperlipidemic mice fed a high-fat diet. Food and Function 11: 6818-6833. https://doi.org/10.1039/d0fo00436g

Hamer, H.M., Jonkers, D., Venema, K., Vanhoutvin, S., Troost, F.J. and Brummer, R.J., 2008. Review article: the role of butyrate on colonic function. Alimentary Pharmacology and Therapeutics 27: 104-119. https://doi.org/10.1111/j.1365-2036.2007.03562.x

Han, B., Baruah, K., Cox, E., Vanrompay, D. and Bossier, P., 2020. Structure-functional activity relationship of beta-glucans from the perspective of immunomodulation: a mini-review. Frontiers in Immunology 11: 658. https://doi.org/10.3389/fimmu.2020.00658

Jayachandran, M., Chen, J., Chung, S.S.M. and Xu, B., 2018. A critical review on the impacts of beta-glucans on gut microbiota and human health. Journal of Nutritional Biochemistry 61: 101-110. https:// doi.org/10.1016/j.jnutbio.2018.06.010

Jayachandran, M., Xiao, J. and Xu, B., 2017. A critical review on health promoting benefits of edible mushrooms through gut microbiota. International Journal of Molecular Sciences 18: 1934. https://doi. org/10.3390/ijms18091934

Jin, M., Zhang, H., Wang, J., Shao, D., Yang, H., Huang, Q., Shi, J., $\mathrm{Xu}, \mathrm{C}$. and Zhao, K., 2019. Response of intestinal metabolome to polysaccharides from mycelia of Ganoderma lucidum. International Journal of Biological Macromolecules 122: 723-731. https://doi. org/10.1016/j.ijbiomac.2018.10.224

Khan, I., Huang, G., Li, X.A., Liao, W., Leong, W.K., Xia, W., Bian, X., Wu, J. and Hsiao, W.L.W., 2019. Mushroom polysaccharides and jiaogulan saponins exert cancer preventive effects by shaping the gut microbiota and microenvironment in $\mathrm{Apc}(\mathrm{Min} /+)$ mice. Pharmacological Research 148: 104448. https://doi.org/10.1016/j. phrs.2019.104448

Kilua, A., Han, K.H. and Fukushima, M., 2020. Effect of polyphenols isolated from purple sweet potato (Ipomoea batatas cv. Ayamurasaki) on the microbiota and the biomarker of colonic fermentation in rats fed with cellulose or inulin. Food and Function 11: 10182-10192. https://doi.org/10.1039/d0fo02111c

Lamichhane, S., Yde, C.C., Jensen, H.M., Morovic, W., Hibberd, A.A., Ouwehand, A.C., Saarinen, M.T., Forssten, S.D., Wiebe, L., Marcussen, J., Bertelsen, K., Meier, S., Young, J.F. and Bertram, H.C., 2018. Metabolic fate of (13)c-labeled polydextrose and impact on the gut microbiome: a triple-phase study in a colon simulator. Journal of Proteome Research 17: 1041-1053. https://doi.org/10.1021/acs. jproteome.7b00683
Larsen, N., Bussolo de Souza, C., Krych, L., Barbosa Cahu, T., Wiese, M., Kot, W., Hansen, K.M., Blennow, A., Venema, K. and Jespersen, L., 2019. Potential of pectins to beneficially modulate the gut microbiota depends on their structural properties. Frontiers in Microbiology 10: 223. https://doi.org/10.3389/fmicb.2019.00223

Lavelle, A. and Sokol, H., 2020. Gut microbiota-derived metabolites as key actors in inflammatory bowel disease. Nature Reviews Gastroenterology and Hepatology 17: 223-237. https://doi. org/10.1038/s41575-019-0258-Z

Li, L., Guo, W.L., Zhang, W., Xu, J.X., Qian, M., Bai, W.D., Zhang, Y.Y., Rao, P.F., Ni, L. and Lv, X.C., 2019a. Grifola frondosa polysaccharides ameliorate lipid metabolic disorders and gut microbiota dysbiosis in high-fat diet fed rats. Food and Function 10: 2560-2572. https:// doi.org/10.1039/c9fo00075e

Li, X., Zeng, F., Huang, Y. and Liu, B., 2019b. The positive effects of Grifola frondosa heteropolysaccharide on NAFLD and regulation of the gut microbiota. International Journal of Molecular Sciences 20: 5302. https://doi.org/10.3390/ijms20215302

Liu, Y., Tang, Q., Yang, Y., Zhou, S., Wu, D., Tang, C., Zhang, Z., Yan, M., Feng, J. and Zhang, J.S., 2017. Characterization of polysaccharides from the fruiting bodies of two species of genus Ganoderma (Agaricomycetes) and determination of water-soluble beta-d-glucan using high-performance liquid chromatography. International Journal of Medical Mushrooms 19: 75-85. https:// doi.org/10.1615/IntJMedMushrooms.v19.i1.80

Long, C., Rosch, C., de Vries, S., Schols, H. and Venema, K., 2020. Cellulase and alkaline treatment improve intestinal microbial degradation of recalcitrant fibers of rapeseed meal in pigs. Journal of Agricultural and Food Chemistry 68: 11011-11025. https://doi. org/10.1021/acs.jafc.0c03618

MacFabe, D.F., 2015. Enteric short-chain fatty acids: microbial messengers of metabolism, mitochondria, and mind: implications in autism spectrum disorders. Microbial Ecology in Health and Disease 26: 28177. https://doi.org/10.3402/mehd.v26.28177

Macfarlane, G.T. and Macfarlane, S., 2012. Bacteria, colonic fermentation, and gastrointestinal health. Journal of AOAC International 95: 50-60. https://doi.org/10.5740/jaoacint.sge macfarlane

Martel, J., Ojcius, D.M., Chang, C.J., Lin, C.S., Lu, C.C., Ko, Y.F., Tseng, S.F., Lai, H.C. and Young, J.D., 2017. Anti-obesogenic and antidiabetic effects of plants and mushrooms. Nature Reviews Endocrinology 13: 149-160. https://doi.org/10.1038/nrendo.2016.142

Martina, A., Felis, G.E., Corradi, M., Maffeis, C., Torriani, S. and Venema, K., 2019. Effects of functional pasta ingredients on different gut microbiota as revealed by TIM-2 in vitro model of the proximal colon. Beneficial Microbes 10: 301-313. https://doi.org/10.3920/ BM2018.0088

Marzullo, P., Di Renzo, L., Pugliese, G., De Siena, M., Barrea, L., Muscogiuri, G., Colao, A., Savastano, S., Obesity Programs of nutrition, E.R. and Assessment, G., 2020. From obesity through gut microbiota to cardiovascular diseases: a dangerous journey. International Journal of Obesity Supplements 10: 35-49. https:// doi.org/10.1038/s41367-020-0017-1 
Matijasic, M., Mestrovic, T., Paljetak, H.C., Peric, M., Baresic, A. and Verbanac, D., 2020. Gut microbiota beyond bacteria-mycobiome, virome, archaeome, and eukaryotic parasites in IBD. International Journal of Molecular Sciences 21: 2668. https://doi.org/10.3390/ ijms 21082668

Miguez, B., Vila, C., Venema, K., Parajo, J.C. and Alonso, J.L., 2020a. Potential of high- and low-acetylated galactoglucomannooligosaccharides as modulators of the microbiota composition and their activity: a comparison using the in vitro model of the human colon TIM-2. Journal of Agricultural and Food Chemistry 68: 7617-7629. https://doi.org/10.1021/acs.jafc.0c02225

Miguez, B., Vila, C., Venema, K., Parajo, J.C. and Alonso, J.L., 2020 b. Prebiotic effects of pectooligosaccharides obtained from lemon peel on the microbiota from elderly donors using an in vitro continuous colon model (TIM-2). Food and Function 11: 9984-9999. https:// doi.org/10.1039/d0fo01848a

Minekus, M., Alminger, M., Alvito, P., Ballance, S., Bohn, T., Bourlieu, C., Carriere, F., Boutrou, R., Corredig, M., Dupont, D., Dufour, C., Egger, L., Golding, M., Karakaya, S., Kirkhus, B., Le Feunteun, S., Lesmes, U., Macierzanka, A., Mackie, A., Marze, S., McClements, D.J., Menard, O., Recio, I., Santos, C.N., Singh, R.P., Vegarud, G.E., Wickham, M.S., Weitschies, W. and Brodkorb, A., 2014. A standardised static in vitro digestion method suitable for food - an international consensus. Food and Function 5: 1113-1124. https:// doi.org/10.1039/c3fo60702j

Minekus, M., Marteau, P., Havenaar, R. and Huis in't Veld, J.H.J., 1995. A multicompartmental dynamic computer-controlled model simulating the stomach and small intestine. Alternatives to Laboratory Animals 23: 197-209.

Minekus, M., Smeets-Peeters, M., Bernalier, A., Marol-Bonnin, S., Havenaar, R., Marteau, P., Alric, M., Fonty, G. and Huis in't Veld, J.H., 1999. A computer-controlled system to simulate conditions of the large intestine with peristaltic mixing, water absorption and absorption of fermentation products. Applied Microbiology and Biotechnology 53: 108-114. https://doi.org/10.1007/s002530051622

Mitsou, E.K., Saxami, G., Stamoulou, E., Kerezoudi, E., Terzi, E., Koutrotsios, G., Bekiaris, G., Zervakis, G.I., Mountzouris, K.C., Pletsa, V. and Kyriacou, A., 2020. Effects of rich in beta-glucans edible mushrooms on aging gut microbiota characteristics: an in vitro study. Molecules 25: 2806. https://doi.org/10.3390/ molecules25122806

Moya, A. and Ferrer, M., 2016. Functional redundancy-induced stability of gut microbiota subjected to disturbance. Trends in Microbiology 24: 402-413. https://doi.org/10.1016/j.tim.2016.02.002

Pan, D., Wang, L., Chen, C., Teng, B., Wang, C., Xu, Z., Hu, B. and Zhou, P., 2012. Structure characterization of a novel neutral polysaccharide isolated from Ganoderma lucidum fruiting bodies. Food Chemistry 135: 1097-1103. https://doi.org/10.1016/j.foodchem.2012.05.071

Pan, Y., Wan, X., Zeng, F., Zhong, R., Guo, W., Lv, X.C., Zhao, C. and Liu, B., 2020. Regulatory effect of Grifola frondosa extract rich in polysaccharides and organic acids on glycolipid metabolism and gut microbiota in rats. International Journal of Biological Macromolecules 155: 1030-1039. https://doi.org/10.1016/j. ijbiomac.2019.11.067
Pan, Y.Y., Zeng, F., Guo, W.L., Li, T.T., Jia, R.B., Huang, Z.R., Lv, X.C., Zhang, J. and Liu, B., 2018. Effect of Grifola frondosa 95\% ethanol extract on lipid metabolism and gut microbiota composition in high-fat diet-fed rats. Food and Function 9: 6268-6278. https://doi. org/10.1039/c8fo01116h

Petersen, E.B.M., Skov, L., Thyssen, J.P. and Jensen, P., 2019. Role of the gut microbiota in atopic dermatitis: a systematic review. Acta Dermato-Venereologica 99: 5-11. https://doi.org/10.2340/000155553008

Reider, S.J., Moosmang, S., Tragust, J., Trgovec-Greif, L., Tragust, S., Perschy, L., Przysiecki, N., Sturm, S., Tilg, H., Stuppner, H., Rattei, T. and Moschen, A.R., 2020. Prebiotic effects of partially hydrolyzed guar gum on the composition and function of the human microbiota-results from the PAGODA trial. Nutrients 12: 1257. https://doi.org/10.3390/nu12051257

Reyes-Castillo, Z., Valdes-Miramontes, E., Llamas-Covarrubias, M. and Munoz-Valle, J.F., 2021. Troublesome friends within us: the role of gut microbiota on rheumatoid arthritis etiopathogenesis and its clinical and therapeutic relevance. Clinical and Experimental Medicine 21: 1-13. https://doi.org/10.1007/s10238-020-00647-y

Robinson, J., Anike, F.N., Willis, W. and Isikhuemhen, O.S., 2018. Medicinal mushrooms supplements alter chicken intestinal microbiome. International Journal of Medicinal Mushrooms 20: 685-693. https://doi.org/10.1615/IntJMedMushrooms.2018026969

Ruthes, A.C., Smiderle, F.R. and Iacomini, M., 2016. Mushroom heteropolysaccharides: a review on their sources, structure and biological effects. Carbohydrate Polymers 136: 358-375. https:// doi.org/10.1016/j.carbpol.2015.08.061

Sameer Kumar, S., Pravamayee, A., Jigni, M., Bighneswar, B., Kumanand, T. and Hrudaynath, T., 2016. Mushroom polysaccharides as potential prebiotics with their antitumor and immunomodulating properties: a review. Bioactive Carbohydrates and Dietary Fibre 7: 1-14. https://doi.org/10.1016/j.bcdf.2015.11.001

Sayago-Ayerdi, S.G., Zamora-Gasga, V.M. and Venema, K., 2019. Prebiotic effect of predigested mango peel on gut microbiota assessed in a dynamic in vitro model of the human colon (TIM-2). Food Research International 118: 89-95. https://doi.org/10.1016/j. foodres.2017.12.024

Shang, Q., Liu, H., Wu, D., Mahfuz, S. and Piao, X., 2021. Source of fiber influences growth, immune responses, gut barrier function and microbiota in weaned piglets fed antibiotic-free diets. Animal Nutrition 7: 315-325.

Shang, Q.H., Liu, S.J., He, T.F., Liu, H.S., Mahfuz, S., Ma, X.K. and Piao, X.S., 2020. Effects of wheat bran in comparison to antibiotics on growth performance, intestinal immunity, barrier function, and microbial composition in broiler chickens. Poultry Science 99: 4929-4938. https://doi.org/10.1016/j.psj.2020.06.031

Su, J., Li, D., Chen, Q., Li, M., Su, L., Luo, T., Liang, D., Lai, G., Shuai, O., Jiao, C., Wu, Q., Xie, Y. and Zhou, X., 2018. Anti-breast cancer enhancement of a polysaccharide from spore of Ganoderma lucidum with paclitaxel: suppression on tumor metabolism with gut microbiota reshaping. Frontiers in Microbiology 9: 3099. https:// doi.org/10.3389/fmicb.2018.03099

Thursby, E. and Juge, N., 2017. Introduction to the human gut microbiota. Biochemical Journal 474: 1823-1836. https://doi. org/10.1042/BCJ20160510 
Tong, A.J., Hu, R.K., Wu, L.X., Lv, X.C., Li, X., Zhao, L.N. and Liu, B., 2020. Ganoderma polysaccharide and chitosan synergistically ameliorate lipid metabolic disorders and modulate gut microbiota composition in high fat diet-fed golden hamsters. Journal of Food Biochemistry 44: e13109. https://doi.org/10.1111/jfbc.13109

Topping, D.L. and Clifton, P.M., 2001. Short-chain fatty acids and human colonic function: roles of resistant starch and nonstarch polysaccharides. Physiological Reviews 81: 1031-1064. https://doi. org/10.1152/physrev.2001.81.3.1031

Vemuri, R., Shankar, E.M., Chieppa, M., Eri, R. and Kavanagh, K., 2020. Beyond just bacteria: functional biomes in the gut ecosystem including virome, mycobiome, archaeome and helminths. Microorganisms 8: 483. https://doi.org/10.3390/ microorganisms 8040483

Venema, K., 2015. The TNO In Vitro Model of the Colon (TIM-2). In: Verhoeckx, K., Cotter, P., Lopez-Exposito, I., Kleiveland, C., Lea, T., Mackie, A., Requena, T., Swiatecka, D. and Wichers, H. (eds.) The impact of food bioactives on health: in vitro and ex vivo models. Springer, Cham, Switzerland, pp. 293-304. https://doi. org/10.1007/978-3-319-16104-4_26

Venema, K., Verhoeven, J., Verbruggen, S. and Keller, D., 2020. Xylooligosaccharides from sugarcane show prebiotic potential in a dynamic computer-controlled in vitro model of the adult human large intestine. Beneficial Microbes 11: 191-200. https://doi. org/10.3920/BM2019.0159

Verduci, E., Mameli, C., Amatruda, M., Petitti, A., Vizzuso, S., El Assadi, F., Zuccotti, G., Alabduljabbar, S. and Terranegra, A., 2020. Early nutrition and risk of type 1 diabetes: the role of gut microbiota. Frontiers in Nutrition 7: 612377. https://doi.org/10.3389/ fnut.2020.612377

Wachtel-Galor, S., Yuen, J., Buswell, J.A. and Benzie, I.F.F., 2011. Ganoderma lucidum (Lingzhi or Reishi): a medicinal mushroom. In: Benzie, I.F.F. and Wachtel-Galor, S. (eds.), Herbal medicine: biomolecular and clinical aspects. CRC Press, Boca Raton, FL, USA, pp. 175-200.
Wang, Y., Liu, Y., Yu, H., Zhou, S., Zhang, Z., Wu, D., Yan, M., Tang, Q. and Zhang, J., 2017. Structural characterization and immunoenhancing activity of a highly branched water-soluble beta-glucan from the spores of Ganoderma lucidum. Carbohydrate Polymers 167: 337-344. https://doi.org/10.1016/j.carbpol.2017.03.016

Wu, X., Cao, J., Li, M., Yao, P., Li, H., Xu, W., Yuan, C., Liu, J., Wang, S., Li, P. and Wang, Y., 2020. An integrated microbiome and metabolomic analysis identifies immunoenhancing features of Ganoderma lucidum spores oil in mice. Pharmacological Research 158: 104937. https://doi.org/10.1016/j.phrs.2020.104937

Xie, J., Liu, Y., Chen, B., Zhang, G., Ou, S., Luo, J. and Peng, X., 2019. Ganoderma lucidum polysaccharide improves rat DSS-induced colitis by altering cecal microbiota and gene expression of colonic epithelial cells. Food and Nutrition Research 63. https://doi. org/10.29219/fnr.v63.1559

Yang, D., Zhou, Z. and Zhang, L., 2019. An overview of fungal glycanbased therapeutics. Progress in Molecular Biology and Translational Science 163: 135-163. https://doi.org/10.1016/bs.pmbts.2019.02.001

Ye, L., Zhang, J., Ye, X., Tang, Q., Liu, Y., Gong, C., Du, X. and Pan, Y., 2008. Structural elucidation of the polysaccharide moiety of a glycopeptide (GLPCW-II) from Ganoderma lucidum fruiting bodies. Carbohydrate Research 343: 746-752. https://doi.org/10.1016/j. carres.2007.12.004

Zhang, Y., Chen, L., Hu, M., Kim, J.J., Lin, R., Xu, J., Fan, L., Qi, Y., Wang, L., Liu, W., Deng, Y., Si, J. and Chen, S., 2020. Dietary type 2 resistant starch improves systemic inflammation and intestinal permeability by modulating microbiota and metabolites in aged mice on high-fat diet. Aging 12: 9173-9187. https://doi.org/10.18632/ aging.103187

Zhou, R., Liu, Z.K., Zhang, Y.N., Wong, J.H., Ng, T.B. and Liu, F., 2019. Research progress of bioactive proteins from the edible and medicinal mushrooms. Current Protein and Peptide Science 20: 196-219. https://doi.org/10.2174/1389203719666180613090710 